\title{
Tratamiento del dolor a través de compresas de calor en el Nuevo Reino de Granada durante los siglos XVIII-XIX
}

\author{
Treatment of pain through heat compresses in the New \\ Kingdom of Granada during the 18th-19th centuries \\ Dr. Eduardo Tuta-Quintero, ${ }^{*, \ddagger}$ Dr. Esteban García-Espitia, ${ }^{\ddagger}$ \\ Dr. Julio César Martínez-Lozano,*,‡ Dr. Eduardo Mazzei-Strocchia, ${ }^{\ddagger}$ \\ Dr. Ignacio Briceño-Balcázar,*,‡ Dra. Marta León-Delgado ${ }^{\ddagger}$
}

Citar como: Tuta-Quintero E, García-Espitia E, Martínez-Lozano JC, Mazzei-Strocchia E, Briceño-Balcázar I, LeónDelgado M. Tratamiento del dolor a través de compresas de calor en el Nuevo Reino de Granada durante los siglos XVIII-XIX. Rev Mex Anestesiol. 2021; 44 (3): 237-240. https://dx.doi.org/10.35366/99673

RESUMEN. Introducción: Manuscritos médicos neogranadinos permitieron la divulgación y conservación de conocimientos y prácticas médicas entre los siglos XVIII y XIX, estos escritos están basados en la administración de agentes medicinales, usados con el fin de atenuar síntomas generados por múltiples enfermedades. Objetivo: Contextualizar el manejo del dolor durante el período colonial a través de una receta médica de la época. Simultáneamente, exponer las posibles bases científicas que expliquen su utilidad. Material y métodos: Búsqueda documental en el archivo histórico de la biblioteca Octavio Arizmendi Posada de la Universidad de La Sabana, donde se encontró la receta médica titulada «Reumas, gota y herpes». Resultados: La medicina durante el siglo XVI y XIX en el Virreinato de la Nueva Granada moduló el dolor a través de la aplicación de calor local en la infección por virus del herpes simple siendo efectivo en la reducción de los síntomas. La eficacia de la termoterapia en el alivio del dolor en enfermedades reumatológicas es controversial. Conclusiones: Los manuscritos médicos permiten viajar al pasado para entender las prácticas médicas de nuestros predecesores. En la actualidad, existe evidencia científica que respalda la efectividad de la aplicación de calor local en el manejo del dolor herpético y reumatológico.

\begin{abstract}
Introduction: New Granada medical manuscripts allowed the dissemination and preservation of medical knowledge and practices between the 18th and 19th centuries, these writings are based on the administration of medicinal agents made of plant, animal, mineral, used to attenuate symptoms generated by multiple diseases. Objective: To contextualize pain management during the colonial period through a medical prescription of the time. Simultaneously, expose the possible scientific bases that explain its usefulness. Material and methods: Documentary search in the historical archive of the Octavio Arizmendi Posada library of the University of La Sabana, where the medical prescription entitled «Reumas, gota $y$ herpes» was found. Results: Medicine during the 16th and 19th centuries in the Viceroyalty of New Granada modulated pain through thermotherapy in herpes simplex virus infection, being effective in reducing pain, inflammation and itching. The efficacy of thermotherapy in relieving pain in rheumatological diseases is controversial. Conclusions: Medical manuscripts allow us to travel to the past to understand the medical practices of our predecessors. There is currently scientific evidence supporting the effectiveness of thermotherapy in the management of herpetic and rheumatological pain.
\end{abstract}

\section{INTRODUCCIÓN}

$\mathrm{E}$ l archivo histórico Cipriano Rodríguez Santa María, de la Universidad de La Sabana, cuenta con múltiples manuscritos médicos que datan de comienzos del siglo XVIII. Uno de éstos registra prácticas médicas utilizadas para el tratamiento del dolor en el Nuevo Reino de Granada (actualmente Colombia). El objetivo de este documento es comprender las bases científicas y culturales que sustentaron estos tratamientos, relativamente exitosos, utilizados en ese período histórico.

\section{Archivo histórico Cipriano Rodríguez}

Desde un punto de vista académico, científico y sociocultural, el estudio de la historia de la medicina nos permite comprender el proceso del desarrollo del conocimiento médico desde el Nuevo Reino de Granada a la actual Colombia a partir del 
fondo documental Cipriano Rodríguez Santa María de la Universidad de La Sabana; el sacerdote Cipriano Rodríguez Santa María, donante de este fondo documental payanés que contiene las recetas médicas aludidas, recibió este legado familiar en razón de que su abuelo materno era bisnieto de Tomás Cipriano de Mosquera, cuatro veces presidente de la República entre 1845 y 1867, y uno de los líderes políticos más influyentes de la nación en el siglo XIX ${ }^{(1)}$.

\section{Recorrido en el tiempo de la medicina del dolor}

El dolor es una experiencia propia de los seres vivos, es una expresión fisiológica de una lesión o de un proceso patológico, y puede considerarse como un signo de alarma esencial para un organismo vivo. Esta experiencia sensorial se percibe por la corteza somatosensorial e interactúa con los centros de memoria de cada individuo, impulsando la toma de decisiones frente a una situación potencialmente nociva ${ }^{(2)}$. Durante siglos y a través de diferentes culturas el significado del dolor ha tenido múltiples acepciones, desde castigos divinos hasta ser entendido como un camino a la trascendencia y purificación del alma; para Hipócrates de Cos (460-360 a. C.) era un desequilibrio de los cuatro humores: sangre, flema, bilis amarilla y negra. Filósofos de la talla de Alcmeón de Crotona (566-497 a. C.), Herófilo (335-280 a. C.) y Erasístrato de Ceos (310-250 a. C.) fueron pioneros en asociar el cerebro como posible integrador de las sensaciones. Galeno (130-201 d. C.) tiempo después enfatiza en la importancia del sistema nervioso central y los nervios periféricos como conductores de la sensibilidad, además, menciona que el dolor es reflejo o señal de advertencia de una enfermedad ${ }^{(3)}$.

En el antiguo Egipto, el dolor producto de una enfermedad era asociado con demonios, dioses, fluidos mágicos y espíritus malignos, cuyo tratamiento realizado por chamanes o curanderos estaba enfocado en la extracción de estos demonios a través de magia o ceremonias religiosas ${ }^{(4)}$. Un tratamiento por medio de agentes herbarios era utilizado por antiguas culturas de medio oriente y Europa. El opio (Papaver somniferum) denominado «planta de la alegría» por los Sumerios, conocido por su potente poder analgésico, fue empleado de manera exitosa en el manejo del dolor por múltiples causas ${ }^{(5)}$. Pese a ello, el afamado médico y botánico alemán Jacobus Theodorus Tabernaemontanus (1525-1590) mencionó tiempo después efectos potencialmente mortales, procurando su uso responsable ${ }^{(3)}$. La medicina china tradicional por su parte, desde el año 2600 a. C. implementó la acupuntura, la moxibustión, entre otras prácticas en el manejo del dolor sin importar su causa, esta tradición se extendería a Europa y América desde el siglo XVI al XIX ${ }^{(6)}$. Por otro lado, culturas preincaicas en el Perú empleaban la planta de coca (Erythroxylum coca) con propósitos religiosos y médicos, resaltando este último como un potente anestésico local ${ }^{(4)}$.

La medicina desde siempre ha buscado modular o suprimir el dolor y cada vez se descubren más elementos relacionados con la fisiopatología de éste, lo cual genera una gran variedad de opciones en el momento de la elección de un tratamiento efectivo $^{(7,8)}$. Teorías para describir los mecanismos del dolor se remontan a varios siglos. René Descartes (1596-1650) dio base sólida a la fisiología moderna del dolor, al integrar las terminaciones nerviosas periféricas con el cerebro o «sensorium común», entendiendo a partir de ello la transducción sensorial (nocicepción) y la experiencia perceptiva del dolor ${ }^{(9)}$. Charles Bell (1774-1842) estudió la anatomía y fisiología del sistema nervioso, diferenciando las neuronas motoras, sensitivas y sensoriales. Además, fue reconocido por fundar la teoría de la Especificidad del dolor ${ }^{(5)}$. Posteriormente, el médico francés Francois Magendie enriquece los hallazgos morfofisiológicos previamente mencionados por Bell, de allí nace la ley de BellMagendie del sistema nervioso ${ }^{(9)}$. Diversas teorías y estudios del dolor fueron realizados por Alfred Goldscheider (1858-1935), Wilhelm H. Erb (1840-1921), Ronald Melzack (1929-2019) y Patrick D. Wall (1925-2001), que permitieron el desarrollo del conocimiento actual, enriqueciendo esta área de la medicina ${ }^{(4)}$.

\section{Abordaje del dolor en el Nuevo Reino de Granada, inicios de un tratamiento innovador}

Durante los siglos XVIII y XIX en el Virreinato de la Nueva Granada, la medicina con influencia europea inició tras la llegada del conocimiento médico del viejo mundo. Los médicos estudiaron la ciencia médica en latín en universidades y colegios médicos en Europa; sin embargo, muchos médicos ejercieron en la América Colonial sin una preparación académica adecuada. Las boticas médicas se presentaron como una solución al precario o nulo sistema de salud existente en la época prehispánica. Éstos eran establecimientos manejados por boticarios con el respaldo de órdenes religiosas, eran los responsables de la producción de sustancias medicinales de origen vegetal principalmente, también se encargaban de estudiar nuevas fórmulas y compuestos químicos ${ }^{(10)}$. La Farmacopea Española y la Farmacopea Matritense permitieron la formación integral de médicos y boticarios. Además, nuevos conocimientos en sustancias medicinales nativas otorgados por expediciones botánicas de científicos como José Celestino Mutis mejoraron el arsenal terapéutico en aquel entonces ${ }^{(11,12)}$.

En el siglo XVIII, los conocimientos de anatomía, química y fisiología permitieron el avance del pensamiento científico en la Nueva Granada, facilitando el diagnóstico y la receta de medicamentos de origen animal, mineral o vegetal. El archivo histórico Cipriano Rodríguez Santa María de la Universidad de La Sabana, posee recetas médicas empleadas entre los siglos XVIII y XIX, entre éstas, se conserva una receta utilizada para el tratamiento del dolor causado por el reumatismo, la gota y el herpes (Figura 1), se hace necesario realizar una mirada histórica a dichas enfermedades y comprender las bases científicas desde el conocimiento médico actual que 
permitan entender el manejo del dolor por medio de compresas de calor durante el período colonial.

\section{A continuación, se realiza la transcripción del documento fuente}

Se presenta de manera literal el documento fuente en el que se encuentra consignado el tratamiento o modulación del dolor durante el período colonial, buscando interpretar y comprender racionalmente los medicamentos de nuestros predecesores.

\section{Uso actual de la termoterapia en la infección por virus del herpes simple y otras enfermedades reumatológicas}

Se sigue usando el calor y el frío como terapias locales para el manejo del dolor causado por el herpes simple y varias enfermedades reumatológicas como la osteoartritis, la artritis reumatoide y la gota. Sin embargo, la evidencia es escasa y controversial. El tratamiento de la infección del virus herpes tipo 1 (HSV-1) y tipo 2 (HSV-2) consiste en la administración de antivirales sistémicos principalmente ${ }^{(13)}$. Algunos estudios han evaluado los beneficios clínicos de la termoterapia con calor local, la cual consiste en aplicar calor en el área de piel afectada, mediante un impulso térmico controlado por microchip, manteniendo una temperatura entre $51-53^{\circ} \mathrm{C}$ durante cuatro segundos. Un estudio que incluyó 103 pacientes con HSV-1 comparó la terapia de calor local (cinco veces al día hasta la desaparición de la sintomatología) con el uso de aciclovir tópico (cinco veces al día por cinco días), demostrando que la terapia con calor local fue superior en la reducción de la sintomatología e incluso disminuyó el tiempo de la enfermedad. Sin embargo, la pobre calidad metodológica de este estudio limita sus resultados. Aunque el mecanismo molecular de la terapia física con calor local no es del todo claro, se ha propuesto que la activación de proteínas de shock térmico como la HSP60 que están involucradas en la regulación de proteínas inmunomoduladoras aceleran la respuesta inmune local, contrarrestando la intensidad de la infección por HSV-1 y HSV-2. A su vez, se ha descrito que la terapia con calor puede contribuir a cierto grado de desnaturalización en las células afectadas previniendo la reproducción de nuevos viriones. Adicionalmente, se ha evidenciado que la terapia con calor local puede reducir la sintomatología por su efecto en las neuronas termo-mecanorreceptoras sensitivas aferentes. Otra hipótesis que explica la efectividad de la terapia con calor es la activación del receptor vaniloide, subfamilia 1 (TRPV-1) que causa entrada de calcio en las fibras $\mathrm{C}$ y $\mathrm{A} \delta$, queratinocitos, fibroblastos, células endoteliales y mastocitos. Su función está relacionada con la sensación térmica y la neuroinmunomodulación. El efecto analgésico y antiprurítico están asociados con la inhibición de la inflamación neurogénica y la alteración de la integridad axonal. Además, se ha encontrado que en células

\begin{tabular}{|c|c|}
\hline $\begin{array}{l}\text { De estas tres enfermedades, dise un buen } \\
\text { médico de Madrid qe ha echo muchisimas } \\
\text { curaciones de enfermos, soldados, totalmente } \\
\text { de brazos, piernas y aunos de medios } \\
\text { cuerpos y que varios médicos habiendo } \\
\text { visto esto maravillosos efectos, han ido y } \\
\text { ban, mundando de los medicamentos que } \\
\text { también usaban, sin utilidad alguna. Y es } \\
\text { el que se sigue: Frotese la agua, suficiente, } \\
\text { para vañarse, la parte enferma. Del agua, } \\
\text { (...) la cantidad qe se saca, de una botella, } \\
\text { qe debe estar muy bien tapado, para que no } \\
\text { se desbanezca, y antes de sacarlo de dicha } \\
\text { botella se debe mezclas y rebolber mui buen, } \\
\text { y la que ha de calentar al fuego y luego se } \\
\text { vañarse otra parte, con ella, abriguese todo } \\
\text { lo vañado, con un paño caliente de lino o } \\
\text { algodon, guardandome el enfermo de vientos } \\
\text { y humedades, Y esa curacion q se haga dos } \\
\text { veces en cada dia. }\end{array}$ & 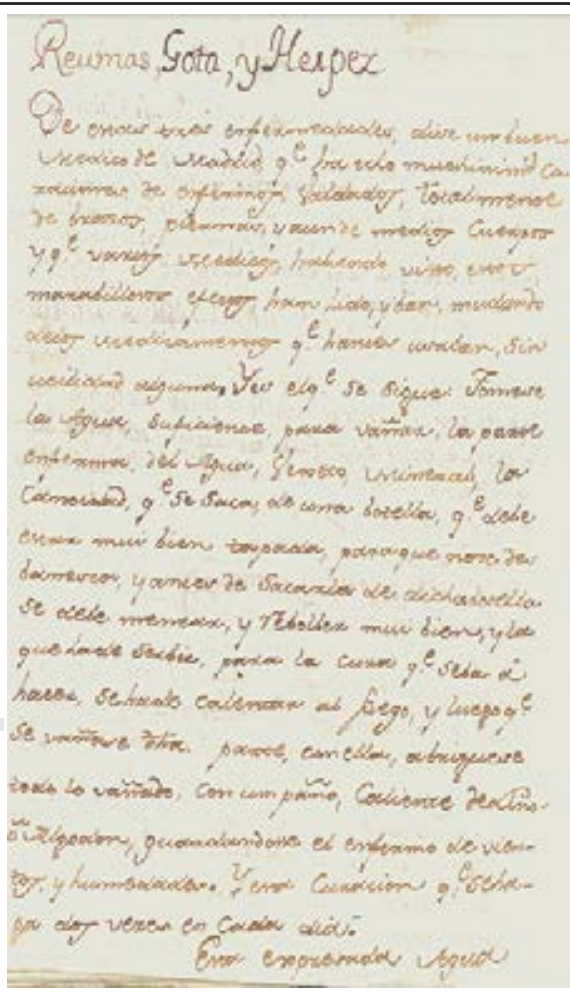 \\
\hline
\end{tabular}

Rheumas, Gota, y herpez

De estas tres enfermedades, dise un buen médico de Madrid qe ha echo muchisimas curaciones de enfermos, soldados, totalmente de brazos, piernas y aunos de medios cuerpos y que varios médicos habiendo visto esto maravillosos efectos, han ido $y$ tambien usaban, sin utilidad alguna. $Y$ es el que se sigue. Frotese la agua, suficiente, para vañarse, la parte enferma. Del agua, (...) la cantidad qe se saca, de una botella, qe debe estar muy bien tapado, para que no vañarse otra parte, con ella, abriguese todo lo vañado, con un paño caliente de lino o algodon, guardandome el enfermo de vientos $y$ humedades, $Y$ esa curacion $q$ se haga dos veces en cada dia.
Figura 1:

Reuma, gota y herpes. Fuente: Archivo Histórico Cipriano Rodríguez Santamaría. Biblioteca Octavio Arizmendi Posada, Universidad de La Sabana. Caja 10, carpeta 2. Disponible en: https://intellectum.unisabana. edu.co/handle/10818/18140 
infectadas por el HSV-1 hay aumento de las proteínas HSP70 Y HSP72 sin la presencia de estrés térmico. En los queratinocitos, la proteína HPS27 tiene un rol fundamental en la inhibición de la liberación de citocinas proinflamatorias, modulando a su vez el factor NF-kB de las células B, explicando el papel de las proteínas de choque de calor en la regulación del dolor y la inflamación ${ }^{(14,15)}$.

Con respecto al uso de la termoterapia en enfermedades reumatológicas, una revisión sistemática en pacientes con artritis reumatoide reportó que el calor y frío local podrían disminuir el dolor en la articulación afectada; sin embargo, dicho tratamiento no detendría la progresión de la enfermedad. Además recomiendan usarla según la necesidad como terapia paliativa y terapia combinada con el ejercicio en pacientes con artritis reumatoide. Se debe tener en cuenta que estas conclusiones se ven limitadas por la pobre calidad metodológica de los estudios disponibles acerca del tema ${ }^{(16)}$. En el caso de la osteoartritis de rodilla una revisión sistemática demostró que los masajes con hielo tuvieron un efecto beneficioso sobre amplitud de movimiento, función y fuerza de la rodilla, además reportan que las bolsas de hidrocoloide disminuyen la inflamación. Las bolsas calientes no tuvieron efectos benéficos sobre el edema y las bolsas de hielo no disminuyeron el dolor en comparación con el control. Por último, concluyen que se necesitan más estudios con mejores metodologías para determinar el papel de la termoterapia en el tratamiento de la osteoartritis de rodilla ${ }^{(17)}$; un estudio que incluyó 150 casos con artritis gotosa, osteoartritis y artritis reumatoide tratados con termoterapia encontró que para los pacientes con artritis gotosa, la terapia con frío local ofreció mayor control del dolor articular comparado con calor local, contrario a los casos con osteoartritis o artritis reumatoide, donde el calor local mostró mayor beneficio para la sintomatología. Este estudio concluye que la termoterapia puede ser usada como adyuvante en sujetos con patologías reumatológicas y que adicionalmente pueden ser un instrumento no invasivo para el diagnóstico de estos tipos de $\operatorname{artritis}^{(18)}$.

\section{CONCLUSIÓN}

La aplicación cutánea de calor durante el período colonial fue una de las medidas utilizadas para el manejo del dolor causado por el herpes simple y otras enfermedades reumáticas. Dicha medida fue de gran utilidad en un período histórico donde la evidencia científica era escasa y no permitía explicar su beneficio, destacando el valor del médico y su juicio para brindar alivio con muy pocos recursos. En la actualidad, estas medidas no farmacológicas siguen siendo parte del manejo del dolor y la inflamación, aunque se recomienda hacer más estudios para conocer completamente su mecanismo de acción y evaluar si son útiles como coadyuvantes al disminuir las dosis de los medicamentos y el tiempo de tratamiento de los fármacos utilizados.

Financiación: El proceso de investigación y publicación fue financiado por las universidades a las cuales están afiliados los autores. Conflicto de intereses: Los autores declaran no tener ningún conflicto de intereses.

\section{REFERENCIAS}

1. Tomás Cipriano de Mosquera - Enciclopedia | Banrepcultural [Internet]. Enciclopedia.banrepcultural.org. 2020 [cited 16 March 2020]. Disponible en: https://enciclopedia.banrepcultural.org/index.php/ Tom\%C3\%A1s_Cipriano_de_Mosquera\#Período\%20Presidencial

2. Garcia-Larrea L, Bastuji H. Pain and consciousness. Prog Neuropsychopharmacol Biol Psychiatry. 2018;87:193-199.

3. Bonica JJ. History of pain concepts and pain therapy. Mt Sinai J Med. 1991;58:191-202.

4. Sabatowski R, Schafer D, Kasper SM, Brunsch H, Radbruch L. Pain treatment: a historical overview. Curr Pharm Des. 2004;10:701-716.

5. El-Ansary M. History of pain relief by ancient Egyptians. Middle East J Anesthesiol. 1989;10:99-105.

6. Zhuang Y, Xing JJ, Li J, Zeng BY, Liang FR. History of acupuncture research. Int Rev Neurobiol. 2013;111:1-23.

7. Mitchell SAT, Majuta LA, Mantyh PW. New Insights in Understanding and Treating Bone Fracture Pain. Curr Osteoporos Rep. 2018;16:325-332.

8. Schneiderhan J, Clauw D, Schwenk TL. Primary care of patients with chronic pain. JAMA. 2017;317:2367-2368.

9. Moayedi M, Davis KD. Theories of pain: from specificity to gate control. J Neurophysiol. 2013;109:5-12.

10. Pita-Pico R. Las boticas en el Nuevo Reino de Granada a finales del período colonial: el lento camino hacia la modernidad. Rev Med. 2015;37:223-241.

11. Tuta-Quintero E, Uribe-Vergara J, Martínez-Lozano JC, Mora-Karam C, Gómez-Gutiérrez A, Briceño-Balcázar I. El guaco: un agente vegetal utilizado en el Nuevo Reino de Granada contra los síntomas generados por afecciones del sistema músculo-esquelético. Rev Colomb Reumatol. 2021;28:5256.

12. Escobar-Robledo M, Briceño-Balcázar I, Tuta-Quintero E, HernándezPardo A, Martínez-Lozano JC, Osorio-Ruiz AM. Manejo médico de apostemas mamarios durante el siglo XVIII en el Nuevo Reino de Granada. Rev Senol Patol Mamar. 2020. https://doi.org/10.1016/j. senol.2020.11.003

13. Arduino PG, Porter SR. Oral and perioral herpes simplex virus type 1 (HSV-1) infection: review of its management. Oral Dis. 2006;12:254-270.

14. Schlippe G, Voss W, Brenn LC. Application and tolerability of Herpotherm $\left({ }^{\circledR}\right)$ in the treatment of genital herpes. Clin Cosmet Investig Dermatol. 2013;6:163-166.

15. Wohlrab J, Vob F, Müller C, Brenn LC. The use of local concentrated heat versus topical acyclovir for a herpes labialis outbreak: results of a pilot study under real life conditions. Clin Cosmet Investig Dermatol. 2013;6:263-271.

16. Welch V, Brosseau L, Casimiro L, Judd M, Shea B, Tugwell P, et al. Thermotherapy for treating rheumatoid arthritis. Cochrane Database Syst Rev [Internet]. 2002. Available in: http://doi.wiley. com/10.1002/14651858.CD00282

17. Brosseau L, Yonge KA, Robinson V, Marchand S, Judd M, Wells G, et al. Thermotherapy for treatment of osteoarthritis. Cochrane Database Syst Rev. 2003;2003:CD004522.

18. Schlesinger N. Response to application of ice may help differentiate between gouty arthritis and other inflammatory arthritides. J Clin Rheumatol. 2006;12:275-276. 